was stirred at room temperature and the oxide went into solution within $10 \mathrm{~min}$. After 45 min, the resulting orange solution was cooled and triethylamine $(40 \mathrm{ml})$ was added, giving colorless gelatinous precipitates. The mixture was evaporated under vacuum to leave a gel. The residue was triturated with boiling $99 \%$ aqueous ethanol $(200 \mathrm{ml})$ and the mixture was allowed to stand at room temperature for $2 \mathrm{hr}$ and then in a refrigerator for $2 \mathrm{hr}$. The insoluble solid was filtered off, washed with $99 \%$ aqueous ethanol, and dried over conc. $\mathrm{H}_{2} \mathrm{SO}_{4}$ at room temperature and $18 \mathrm{~mm} \mathrm{Hg}$ for $15 \mathrm{hr}$ to give an anhydrous sample of III, mp $239-240^{\circ}$ (decomp.), shown to be pure by means of PPC. Yield, $17.8 \mathrm{~g}(83 \%)$. Recrystallization from $99 \%$ aqueous ethanol and drying over $\mathrm{P}_{2} \mathrm{O}_{5}$ at $110^{\circ}$ and $3 \mathrm{~mm} \mathrm{Hg}$ for $3 \mathrm{hr}$ furnished an analytical sample as colorless needles, $\mathrm{mp} 240-241^{\circ}$ (decomp.) [lit. ${ }^{4)} \mathrm{mp} 176-178^{\circ}$ (decomp.)]; $[\alpha]_{\mathrm{D}}^{19}-51.4^{\circ}(c=1.00, l=$ $\left.0.5, \mathrm{H}_{2} \mathrm{O}\right)$; UV $\lambda_{\max }^{95 \% \operatorname{sq} \cdot \mathrm{EtOH}} \mathrm{m} \mu(\varepsilon): 235(40700), 264(7800), 301(2300) ; \lambda_{\max }^{\mathrm{H}_{2} \mathrm{O}}(\mathrm{pH} 1)^{10)} 258(12100) ; \lambda_{\max }^{\mathrm{H}_{2} \mathrm{O}}(\mathrm{pH} 7)^{11)}$ $233(40800), 262(8000), 296(2100) ; \lambda_{\max }^{\mathrm{H}_{2} \mathrm{O}}(\mathrm{pH} 13)^{12)} 232(22500), 269(8400), 310(4100)$. Anal. Calcd. for $\mathrm{C}_{13} \mathrm{H}_{17} \mathrm{O}_{5} \mathrm{~N}_{5}: \mathrm{C}, 48.29 ; \mathrm{H}, 5.30 ; \mathrm{N}, 21.66$. Found: $\mathrm{C}, 48.29 ; \mathrm{H}, 5.44 ; \mathrm{N}, 21.86$. Identity of this sample with the one obtained by the earlier method ${ }^{4}$ : was established by mixed melting-point test, PPC, and ultraviolet and IR spectra.

Replacement of the monohydrate $\left(\mathrm{IV} \cdot \mathrm{H}_{2} \mathrm{O}\right)$ by an anhydrous sample in this reaction could not increase the yield of III.

Acknowledgement Thanks are given for financial support through a Grant-in-Aid for Cancer Research (92058, to Professor D. Mizuno) from the Ministry of Education, Japan. We also acknowledge with thanks several conversations with Dr. T. Ueda, Hokkaido University, and with Dr. A. Yamazaki, Ajinomoto Co., Inc.

10) Measured in $0.1 \mathrm{~N} \mathrm{HCl}$.

11) Measured in $0.005 \mathrm{M}$ phosphate buffer.

12) Measured in $0.1 \mathrm{~N} \mathrm{NaOH}$.

\title{
Studies on Phenothiazinyl Radicals
}

\author{
Mamoru Kamiya, Takehiro Mitsui and Yukio Akahori ${ }^{1)}$
}

\author{
Shizuoka College of Pharmacy
}

(Received June 21, 1972)

During the course of numerous studies on phenothiazine radicals, it has been indicated by electron spin resonance (ESR) method that neutral phenothiazinyl radical which is rather unstable can be produced from phenothiazine by several methods. ${ }^{2-4}$ ) However, any work has not been published regarding the electronic absorption spectrum of these radicals. This may be chiefly because, as seen in general, measuring and assigning the spectrum of such unstable radical are seriously prevented from coexistence of various radical decay products. In this note we shall report the electronic absorption spectra of non-substituted and 1-methylsubstituted phenothiazinyl radicals which are generated in degassed dimethylsulfoxide-acetic anhydride mixture. By referring to the ESR spectra in the same systems and by performing theoretical calculations on the electronic transition of the radicals, a brief comment will be given to the assignment of the new absorption maxima in the electronic absorption spectra.

\section{Experimental}

1-Methylphenothiazine was prepared from thionation of the corresponding diarylamine by the method described in the literature. ${ }^{5}$ (mp 136 ${ }^{\circ}$. Anal. Calcd. for $\mathrm{C}_{13} \mathrm{H}_{11} \mathrm{NS}: \mathrm{C}, 73.20 ; \mathrm{H}, 5.20 ; \mathrm{N}, 6.57$. Found:

1) Location: Oshika, Shizuoka-shi.

2) H.J. Shine and E.E. Mach, J. Org. Chemi, 30, 2130 (1965).

3) B.C. Gilbert, P. Hanson, R.O.C. Norman, and B.T. Sutcliffe, Chem. Comm., 1966, 161.

4) Y. Tsujino, Tetrahedron Letters, 1968, 4111.

5) S.P. Massie and P.K. Kadaba, J. Org. Chem., 21, 347 (1956). 
$\mathrm{C}, 73.09 ; \mathrm{H}, 5.21 ; \mathrm{N}, 6.49$.$) . The compounds used were purified by repeated sublimations under a high$ vacuum $\left(\sim 10^{-6} \mathrm{~mm}\right)$. The solvent used for the radical formation was prepared by mixing equimolar amounts of spectro-grade dimethylsulfoxide and acetic anhydride. The radical solutions were formed by dissolving the weighted materials in degassed solvent. The radical solutions were filtered through a glassfilter, and were directly led to a quartz ultraviolet (UV) cell $(10 \mathrm{~mm})$ or a $\mathrm{ESR}$ tube. The spectroscopic measurements were done using a Hitachi ESP-2 spectrophotometer and a JES-3BS-X spectrometer.

The theoretical results were obtained with the semiempirical open-shell SCF method ${ }^{6}$ ) with limited (I Parametrizations are analogous to that reported in a previous paper, ${ }^{7)}$ and are (lenoted in Table I with the theoretical results. The CI calculation was done inchuding 24 singly excited configurations composer of $(\mathrm{i} \rightarrow \mathrm{k})_{\alpha},(\mathrm{i} \rightarrow \mathrm{k})_{\beta}$, and $(\mathrm{i} \rightarrow \mathrm{m})$ and $\left.(\mathrm{m} \rightarrow \mathrm{k}) .^{8}\right)$

TABLE I. Experimental and Theoretical Results of Filectronic Spectra.

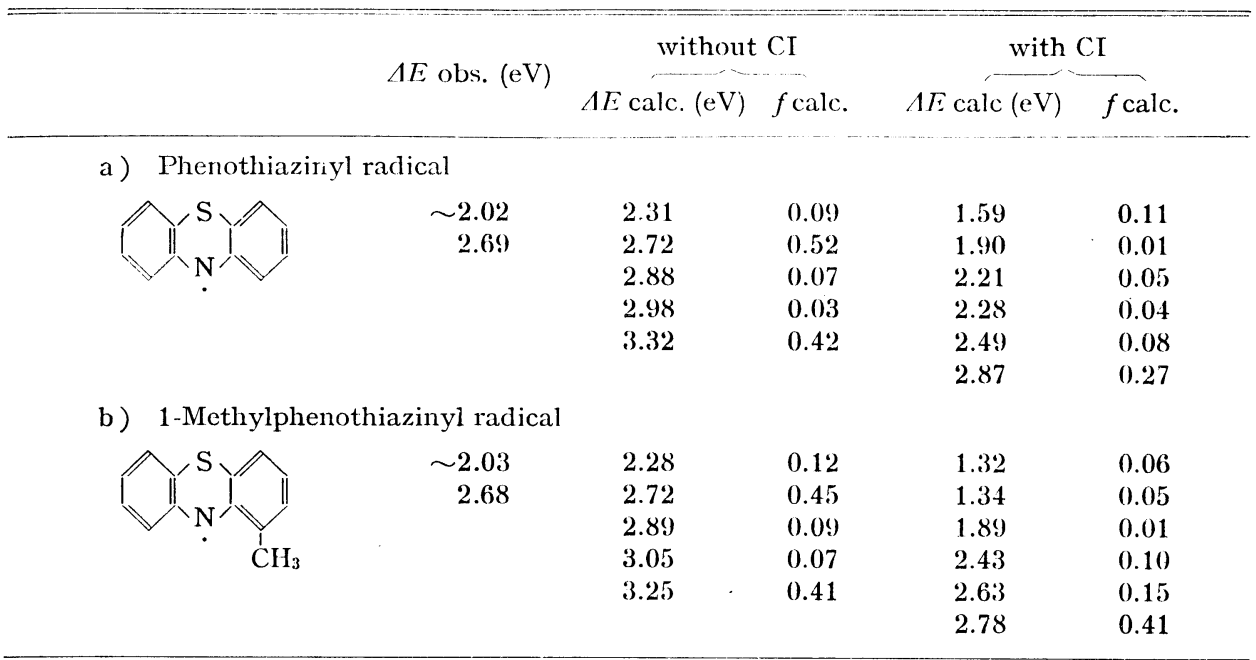

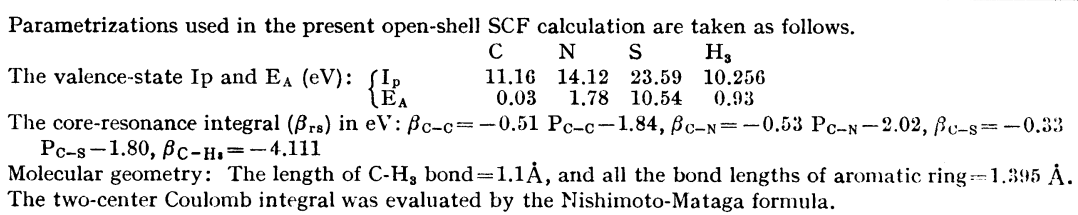

\section{Result}

Fig. 1(a) indicates that new absorption maxima grow in the regions of 462 and $615 \mathrm{~nm}$ as the radical formation proceeds in phenothiazine DMSO- $\mathrm{Ac}_{2} \mathrm{O}$ solution. The longer wavelength band has a $\sim 645$-nm shoulder. Similar changes in the electronic absorption spectrum are also observed in the 1-methylphenothiazine system (Fig. 1(b)); The $\lambda_{\mathrm{nm}}^{\max }$ values of the new absorption bands are 464 and $610 \mathrm{~nm}$, respectively. The absorption spectrum in the region of wavelengths shorter than $\sim 400 \mathrm{~nm}$ had to be excluded from consideration on account of the intense absorption of the parent molecules which would mask the changes in the spectrum. The new absorption maxima characteristic of the two systems were reproduced precisely at a number of the radical formations in which degassings were performed carefully using a high vacuum line, and thus the new absorption maxima appear to be related to the process of the radical formations. In fact, the solutions exhibiting the absorption maxima showed the

6) H.C. Longuet-Higgins and J.A. Pople, Proc. Phys. Soc. (London), A68, 591 (1955).

7) M. Kamiya, Bull. Chem. Soc. Japan, 45, 1589 (1972).

8) $\phi_{\mathrm{m}}=$ half-occupied orbital; $\mathrm{i}=\mathrm{m}-3, \mathrm{~m}-2, \mathrm{~m}-1$ and $\mathrm{k}=\mathrm{m}+1, \mathrm{~m}+2, \mathrm{~m}+3$; The notation of $(\mathrm{i} \rightarrow \mathrm{k})_{\boldsymbol{a}}$ and $(\mathrm{i} \rightarrow \mathrm{k})_{\beta}$ was taken according to the paper of Ishitani and Nagakura (A. Ishitani and S. Nagakura, Theor. Chim. Acta, 4, 236 (1966)). 


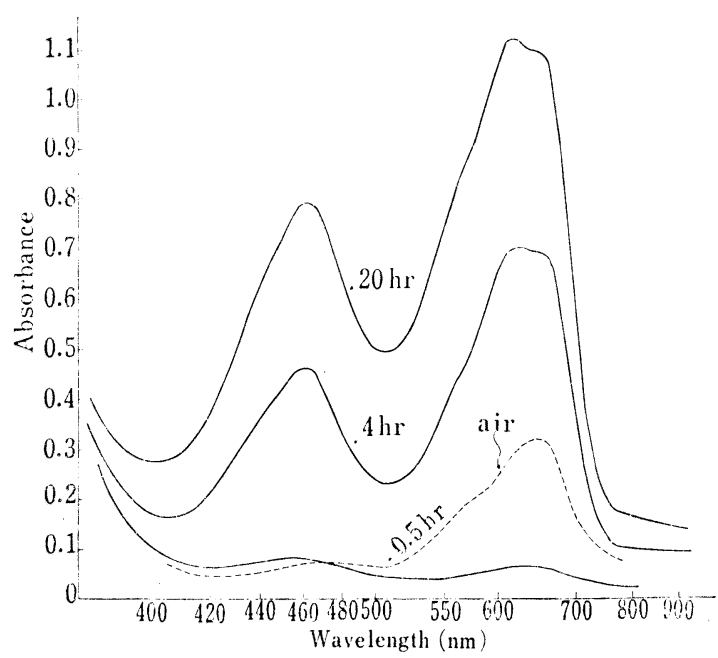

Fig. 1(a). Electronic Spectra Observed in thenothiazine $+\mathrm{DMSO}-\mathrm{Ac}_{2} \mathrm{O}$. conc. of phenothiazine =$1.12 \times 10^{-3} \mathrm{M}$

The dotted absorption curve is that measured $15 \mathrm{hr}$ after air-contact

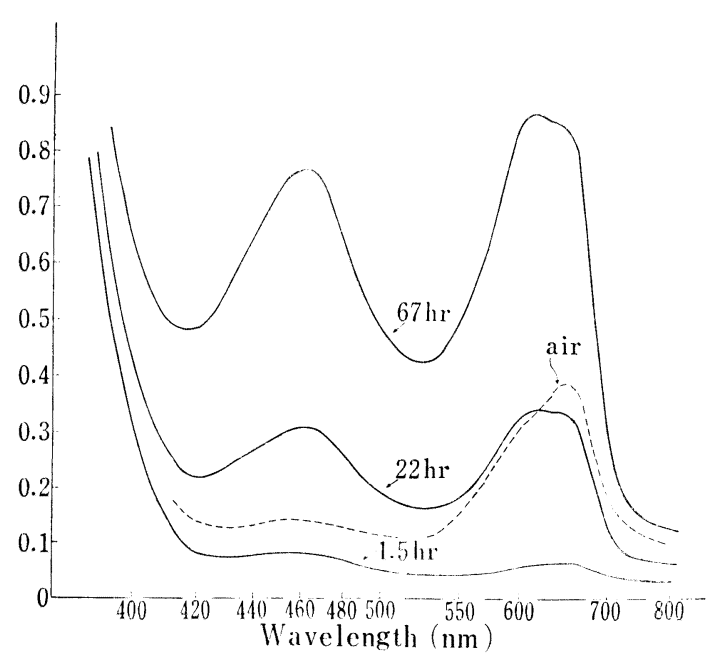

lig. 1(b). Electronic spectra Observed in 1-methylphenothiazine $+\mathrm{DMSO}-\mathrm{AC}_{2} \mathrm{O}$. conc. of 1-methylphenothiazine $=5.13 \times 10^{-3} \mathrm{M}$

The dotted absorption curve is that measured $46 \mathrm{hr}$ after air-contact.

strong ESR spectra as will be mentioned later. The ESR spectrum of non-substituted phenothiazinyl radical has already been reported by several literatures. ${ }^{2-4)}$ The ESR spectrum of 1-methyl-substituted phenothiazinyl radical has been treated here for the first time, and the spectrum is shown in Fig. 2. Though not completely resolved, the ESR spectrum could be analyzed with $a^{\mathrm{N}}=7.0-7.2$ gauss, $a_{3,7}^{\mathrm{H}}=3.5$ gauss, $a_{9}^{\mathrm{H}}=2.2-2.5$ gauss and $a_{2.8}^{\mathrm{H}}=0.7-0.9$ gauss. These values of the hyperfine splitting constants are similar with the values for the non-substituted radical which have been reported. ${ }^{2 \cdot 4)}$

Fig. 3 indicates the decays of the absorbance of the new absorption maxima when the solutions were exposed to air. During the initial course of time, the decay rates of the two absorption maxima in the phenothiazine system were in a same order, but the decay of the 615-nm absorbance stopped much faster than that of the 462-nm absorbance. The solutions which were exposed to air for $15 \mathrm{hr}$ did not show any ESR spectrum of phenothiazinyl radical, but the absorbance of the 615 $\mathrm{nm}$ region retained a considerable value. At this stage, the location of $\lambda_{\mathrm{mn}}^{\max }$ of the longer wavelength band shifted to $\sim 645$ $\mathrm{nm}$. In this region of wavelength, a shoulder was observed at the initial spectrum. Similar changes were found when the solution of 1-methylphenothiazinyl radical was exposed to air. But the decay rate of the new absorption maxima was found to be remarkably slower than that for the phenothiazine system; The solution which was exposed to air for $46 \mathrm{hr}$ did not show the ESR spectrum of the substituted radical, but the electronic absorption spectrum showed a considerable value of absorb-

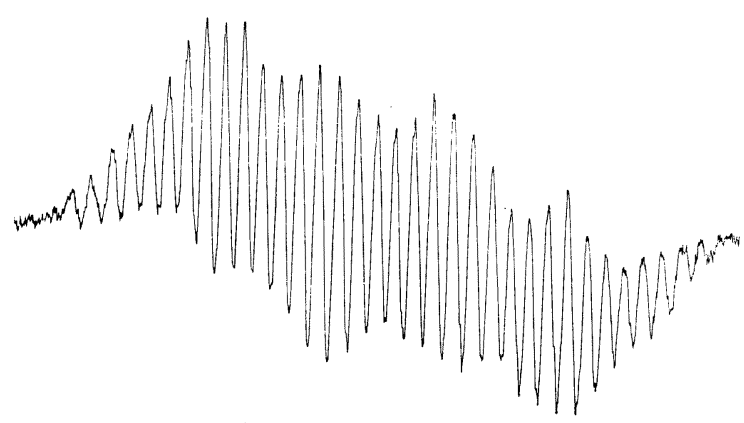

Fig. 2. ESR Spectrum of 1-Methylphenothiazinyl Radical produced in DMSO $+\mathrm{Ac}_{2} \mathrm{O}$ at Room Temperature 
ance in the region of $640-645 \mathrm{~nm}$.

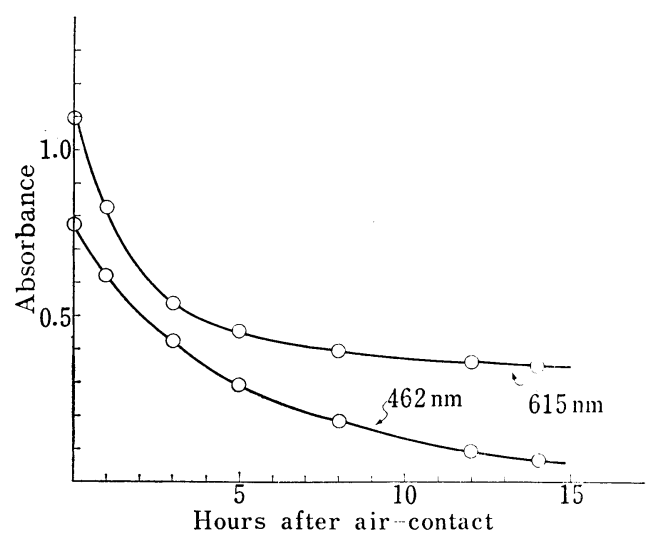

Fig. 3(a). Decay Curves of the New Absorption Maxima on Air-contact (Phenothiazine system)

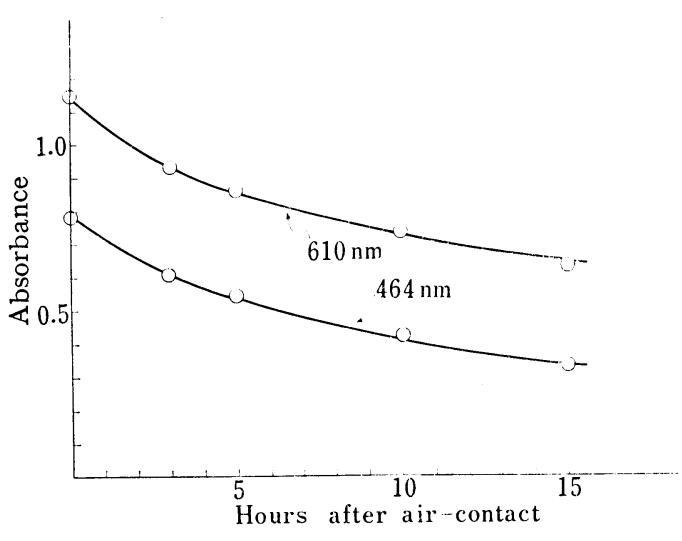

Fig. 3(b). Decay Curves of the New Absorption Maxima on Air-contact (1-methylphenothiazine system)

These experimental results indicate that the longer wavelength band in the spectrum unexposed to air contains the absorption which may be assigned to stable compounds produced through the radical formation. Here, the literatures ${ }^{4,9)}$ report that $1,10^{\prime}$ - and $3,10^{\prime}$-dimers of phenothiazine are readily produced via the phenothiazinyl radical. The solutions which were exposed to air until any ESR spectrum of the radicals could not be detected were submitted to thin-layer chromatography using petroleum ether-ether $(2: 1)$ as developing solvent according to the method of Tsujino. ${ }^{8}$ The $1,10^{\prime}$ - and 3,10'-dimers of phenothiazine were identified by the spots with $R f=0.8-0.9$ and $0.4-0.5$, respectively, in accord with the results by Tsujino. The 3,10'-dimer of 1-methylphenothiazine was also detected by the spot with $R f=$ $0.3-0.4$, but the spot corresponding to the 1,10'-dimer could not be found. The spot with $R f=0.0-0.2$ which would be related to the polymers as radical decay products were also found in the two systems. Thus, we may assign the $640-645 \mathrm{~nm}$ absorption to these by-products. And it is not so unreasonable to assume that the main component is the $3,10^{\prime}$-dimer, because Tsujino reports the highest yield of the dimer from phenothiazine and because the absorption curve in the region of $640-645 \mathrm{~nm}$ is similar at the two systems.

The theoretical data on the electronic transition suggest existence of weak transitions below $-2 \mathrm{eV}$ which might be masked by the intense absorption of the by-products. This trend is promoted by the CI calculation which exerts a great effect to the theoretical data. Nevertheless, the theoretical data both with and without CI indicate that moderately intense transition of the neutral radicals exists just in the region of the shorter wavelength bands which grow on the radical formation.

The effects of photoirradiation to the radical solutions are now under investigation.

Acknowledgement The authors wish to thank Dr. Michiya Itoh for his useful discussion. Thanks are also due to Mr. Takeshi Akiyama for his co-operation and to the members of analytical center for elementary analysis.

9) Y. Tsujino, Nippon Kagaku Zasshi, 90, 304 (1969). 